\title{
Cough cardiopulmonary resuscitation for bradycardia management: good or bad?
}

\author{
Nishkarsh Gupta ${ }^{1}$ and Anju Gupta ${ }^{2}$ \\ ${ }^{I}$ Department of Onco-Anesthesiology and Palliative Medicine, DRBRAIRCH, All India Institute of Medical \\ Sciences, ${ }^{2}$ Department of Anesthesiology and Critical Care, Vardhman Mahavir Medical College and Safdarjung \\ Hospital, New Delhi, India
}

We read an interesting report by Karippacheril et al. [1], on a patient who developed severe bradycardia/asystole during total hip arthroplasty (THR) and responded to the 'cough cardio-pulmonary resuscitation (CPR)'. We are not entirely convinced by the author's conclusions and have some concerns.

Bradycardia $(<50 \mathrm{bpm})$ is common after spinal anesthesia and may in occur in up to $10 \%$ of the patients [2]. The causes of bradycardia after spinal anesthesia are not clear, and some of the reasons include blockade of T1-4 cardiac sympathetic fibers, vasodilatation, and decreased venous return to the right atrium. Furthermore, bradycardia generally occurs within 60 minutes of spinal anesthesia, when the cardiovascular effects of spinal anesthesia and positional changes are maximum. The Bezold-Jarisch reflex is a paradoxical increase in inhibitory neural activity to the heart because of the poorly filled hypercontractile left ventricle and can cause severe bradycardia. It is most likely to occur because of sudden absolute/relative volume depletion, which may occur when the effect of spinal anesthesia is maximum in the initial phases, during extreme positional changes or sudden loss of blood during the procedure.

In the case described by Karippacheril et al. [1], the patient was a healthy young adult who developed severe bradycardia/

Corresponding author: Anju Gupta, M.D.

Department of Anesthesiology and Critical Care, Vardhman Mahavir Medical College and Safdarjung Hospital, New Delhi 110029, India

Tel: +91-9911573371, Fax: +91-011-26163072

Email: dranjugupta2009@rediffmail.com

ORCID: https://orcid.org/0000-0003-1726-1488

Received: June 9, 2019.

Revised: June 17, 2019.

Accepted: June 18, 2019.

Korean J Anesthesiol 2019 October 72(5): 510-511

https://doi.org/10.4097/kja.19252 asystole 90 minutes after spinal anesthesia during THR. They attributed the mechanism to the Bezold-Jarisch reflex. However, there had been no sudden posture changes or blood loss during the procedure. Moreover, if the Bezold-Jarisch reflex was the suspected cause, fluid bolus should have been administered.

Atropine and epinephrine can manage the symptomatic bradycardia leading to hypoperfusion to the heart and brain. Transcutaneous pacing and dopamine are considered in severe cases, when first-line drugs are not effective. As a standard practice, we routinely load a syringe with atropine and epinephrine in patients undergoing surgery under spinal anesthesia. The patient reported here had severe symptomatic bradycardia with probably transient asystole. As a management option, 'cough CPR' was considered initially. The sequence of events of loss of QRS complexes on electrocardiogram (ECG), while the pulse rate tracing and pulse oximeter readings were present, cause confusion. If the corresponding ECG tracings and monitor readings were available, the confusion could be resolved. While the patient was being resuscitated, a mental track of events could not have been kept, and use of screenshots and trends in monitor memory would have been better. Moreover, the flat line protocol was not followed. It is possible that some interference due to the use of electrocautery led to the loss of ECG traces.

A forceful cough may maintain enough blood flow to the brain because of increased intrathoracic pressure and may help the patient maintain consciousness for a few seconds, until arrhythmia is treated. A forced cough may pump up to $700 \mathrm{ml}$ of blood from the extremities, if a healthy volunteer coughs using the total lung capacity [3].

However, in a patient with spinal anesthesia undergoing THR in the lateral position, the effectiveness of cough would be substantially reduced [4]. Moreover, severe bradycardia/asystole is an emergency, and intricacies of the 'cough' maneuver could not have been explained to the patient for an effective CPR. The

(c) This is an open-access article distributed under the terms of the Creative Commons Attribution Non-Commercial License (http://creativecommons.org/ licenses/by-nc/4.0/), which permits unrestricted non-commercial use, distribution, and reproduction in any medium, provided the original work is properly cited. 
American Heart Association has described it to be useful only transiently, for in-hospital situations, in a controlled environment, in a conscious and responsive person [5]. However, its usefulness in the present scenario is doubtful as the authors have mentioned that the patient was 'partially responsive' at the time of the event.

We doubt the usefulness of 'cough CPR' in the patient as a first choice instead of atropine or epinephrine administration, which is usually loaded for all elective cases under spinal anesthesia for any eventuality. Furthermore, the level of the spinal blockade at the time was not mentioned, which may have been higher and may have caused extensive sympathetic blockade, leading to bradycardia. This is evident from the fact that 'cough CPR' did not improve the patient's condition remarkably. 'Cough CPR' caused loss of valuable time and delayed the use of atropine and epinephrine. The patient's condition eventually improved after atropine and epinephrine. A further delay may have been catastrophic. We believe that surgery should be immediately stopped in such a case; the patient should be placed supine; and $100 \%$ oxygen and atropine should be administered. The reported patient remained lateral for some time while cough CPR was attempted.

We do not confer to the authors' suggestion that 'cough CPR' should be attempted routinely in cases of bradycardia under spinal anesthesia.

\section{Conflicts of Interest}

No potential conflict of interest relevant to this article was reported.

\section{Author Contributions}

Nishkarsh Gupta (Writing-original draft; Writing-review \& editing)

Anju Gupta (Resources; Writing-original draft; Writing-review \& editing)

\section{ORCID}

Nishkarsh Gupta, https://orcid.org/0000-0002-8444-2564

Anju Gupta, https://orcid.org/0000-0003-1726-1488

\section{References}

1. Karippacheril JG, Philip A, Ashraf Y. Forced cough for witnessed extreme bradycardia in hip arthroplasty: a maneuver in extremis. Korean J Anesthesiol 2019; 72: 279-80.

2. Lesser JB, Sanborn KV, Valskys R, Kuroda M. Severe bradycardia during spinal and epidural anesthesia recorded by an anesthesia information management system. Anesthesiology 2003; 99: 859-66.

3. LoMauro A, Aliverti A. Blood shift during cough: negligible or significant? Front Physiol 2018; 9: 501.

4. Harrop-Griffiths AW, Ravalia A, Browne DA, Robinson PN. Regional anaesthesia and cough effectiveness. A study in patients undergoing caesarean section. Anaesthesia 1991; 46: 11-3.

5. Cave DM, Gazmuri RJ, Otto CW, Nadkarni VM, Cheng A, Brooks SC, et al. Part 7: CPR techniques and devices: 2010 American Heart Association Guidelines for Cardiopulmonary Resuscitation and Emergency Cardiovascular Care. Circulation 2010; 122(18 Suppl 3): S7208. 